\title{
A simple proof of the Hardy inequality on Carnot groups and for some hypoelliptic families of vector fields
}

\author{
F. Vigneron \\ francois.vigneron@u-pec.fr \\ Univ. Paris-Est Créteil, LAMA, UMR8050 du CNRS \\ 61, avenue du Général de Gaulle, F94010 Créteil, France.
}

August 20, 2019

\begin{abstract}
We give an elementary proof of the classical Hardy inequality on any Carnot group, using only integration by parts and a fine analysis of the commutator structure, which was not deemed possible until now. We also discuss the conditions under which this technique can be generalized to deal with hypo-elliptic families of vector fields, which, in this case, leads to an open problem regarding the symbol properties of the gauge norm.
\end{abstract}

Keywords: Hardy inequality, Carnot group, Stratified algebra, Commutators, Hypoellliptic vector fields, Hörmander bracket condition.

MSC classification: 43A80, 35R03, 42B37.

The classical Hardy inequality [17] on a smooth open domain $\Omega \subset \mathbb{R}^{n}(n \geq 3)$ reads:

$$
\forall f \in H_{0}^{1}(\Omega), \quad \sup _{x_{0} \in \Omega}\left(\int_{\Omega} \frac{|f(x)|^{2}}{\left|x-x_{0}\right|^{2}} d x\right) \leq \frac{4}{(n-2)^{2}} \int_{\Omega}|\nabla f(x)|^{2} d x .
$$

Since L. D'Ambrosio [11] it has been well known that similar inequalities hold on nilpotent groups, but interest on this matter is still high; see e.g. [27], [1], [2].

An important reference on this matter is a recent note by H. Bahouri, C. Fermanian, I. Gallagher [8]. It is dedicated to refined Hardy inequalities on graded Lie groups and relies on constructing a general Littlewood-Paley theory and, as such, involves the machinery of the Fourier transform on groups.

Expanding the generality towards hypoelliptic vector fields, G. Grillo's [15] article contains an inequality that holds for $L^{p}$-norms, without an underlying group structure, and contains weights that allow positive powers of the Carnot-Caratheodory distance on the right-hand side. However, the proof of this generalization involves the whole power of the sub-Riemannian Calderon-Zygmund theory.

Another beautiful reference is the paper by P. Ciatti, M.G. Cowling, F. Ricci [10] that studies these matters on stratified Lie groups, but with the point of view of operator and interpolation theory (see also [21] to highlight some subtleties in this approach).

The main goal of the present paper is to prove a general result on Carnot groups, albeit slightly simpler than those of [8] or [15], by using only elementary techniques: most of the paper relies only on integrations by part and on a fine analysis of the commutator structure. We will occasionally use interpolation techniques, but it is only required here if fractional regularities are sought after.

A Carnot group is a connected, simply connected and nilpotent Lie group $G$ whose Lie algebra $\mathfrak{g}$ admits a stratification, i.e.

$$
\mathfrak{g}=\bigoplus_{j=1}^{m} V_{j} \quad \text { where } \quad\left[V_{1}, V_{j}\right]=V_{j+1}
$$

with $V_{m} \neq\{0\}$ but $\left[V_{1}, V_{m}\right]=\{0\}$. The dimensions will be denoted by $q_{j}=\operatorname{dim} V_{j}$ and $q=\sum q_{j}=$ $\operatorname{dim} \mathfrak{g}$. Given a basis $\left(Y_{\ell}\right)_{\ell=1, \ldots, q}$ of $\mathfrak{g}$ adapted to the stratification each index $i \in\{1, \ldots, q\}$ can be 
associated to a unique weight $\omega_{\ell} \in\{1, \ldots, m\}$ such that $Y_{\ell} \in V_{\omega_{\ell}}$, namely

$$
\omega_{\ell}=j \quad \text { for } \quad n_{j-1}<\ell \leq n_{j}
$$

where $n_{0}=0$ and $n_{j}=n_{j-1}+q_{j}$ for $j=1, \ldots, m$ is the sequence of cumulative dimensions. Note that $n_{1}=q_{1}$ and $n_{m}=q$. The horizontal derivatives are the derivatives in the first layer (see $\S 1.5$ below) and they are collected together in the following notation:

$$
\nabla_{G} f=\left(Y_{1}^{L} f, \ldots, Y_{n_{1}}^{L} f\right) .
$$

The stratification hypothesis ensures that each derivative $Y_{i} f$ can be expressed as at most $\omega_{i}-1$ commutators of horizontal derivatives. The homogeneous dimension is the integer

$$
Q=\sum_{j=1}^{m} j q_{j}=\sum_{\ell=1}^{q} \omega_{\ell}
$$

For $k \in \mathbb{N}$, the Sobolev space $H^{k}(G)$ is the subspace of functions $\phi \in L^{2}(G)$ such that $\nabla_{G}^{\alpha} \phi \in L^{2}(G)$ for any multi-index $\alpha$ of length $|\alpha| \leq k$. Fractional spaces can, for example, be defined by interpolation. The main result that we intend to prove here is the following.

Theorem 1 Let $G$ be a Carnot group and $\|\cdot\|$ any homogeneous pseudo-norm equivalent to the CarnotCaratheodory distance to the origin. Then, for any real $s$ with $0 \leq s<Q / 2$, there exists a constant $C_{s}>0$ such that:

$$
\int_{G} \frac{|f(g)|^{2}}{\|g\|_{G}^{2 s}} d g \leq C_{s}\|f\|_{H^{s}(G)}^{2}
$$

for any function $f \in H^{s}(G)$.

A similar Hardy inequality was proved by the author in [29] for families of vector fields that satisfy a Hörmander bracket condition of step 2; the proof was based on the ideas of [4] and [7] but was never published independently. This result was part of a broader study [5], [6], [23], [28] aiming at characterizing the traces of Sobolev spaces on the Heisenberg group, along hypersurfaces with nondegenerate characteristic points. Here, instead, we concentrate (except in §3) on the case of stratified groups, but without restrictions on the step $m$ of the stratification.

The mathematical literature already contains numerous Hardy-type inequalities either on the Heisenberg group, for the $p$-sub-Laplacian, for Grushin-type operators and $H$-type groups (see e.g. [11], [20]). Sometimes (e.g. in [14], [24], [12]), a weight is introduced in the left-hand side that vanishes along the center of the group, i.e. along the (most) sub-elliptic direction. For example, [14] contains the following inequality on the Heisenberg group $\mathbb{H}_{n} \simeq \mathbb{C}^{n} \times \mathbb{R}$ :

$$
\int_{\mathbb{H}_{n}} \frac{|f(x)|^{2}}{d_{\mathbb{H}_{n}}(x, 0)^{2}} \Phi(x) d x \leq A \sum_{j=1}^{n}\left(\left\|X_{j} f\right\|_{L^{2}}^{2}+\left\|Y_{j} f\right\|_{L^{2}}^{2}\right)+B\|f\|_{L^{2}}^{2}
$$

where $\left(X_{j}, Y_{j}\right)$ are a basis of the first layer of the stratification and $d_{\mathbb{H}_{n}}((z, t), 0) \simeq \sqrt[4]{|z|^{4}+t^{2}}$ is the gauge distance and $\Phi$ is a cut-off function that vanishes along the center $z=0$ :

$$
\Phi(z, t)=\frac{|z|^{2}}{\sqrt{|z|^{4}+t^{2}}}
$$

A secondary goal of this article is to show that such a cut-off is usually not necessary.

The core of our proof of theorem 1 (see $\S 2.5$ ) consists in an integration by part against the radial field (the infinitesimal generator of dilations). The radial field can be expressed in terms of the leftinvariant vector fields but, as all strata are involved, this first step puts $m-s$ too many derivatives on the function. Next, one uses the commutator structure of the left-invariant fields to carefully backtrack 
all but one derivative and let them act instead on the coefficients of the radial field. This step requires that those coefficients have symbol-like properties. One can then conclude by an iterative process that reduces the Hardy inequality with weight $\|g\|_{G}^{-s}$ to the one with weight $\|g\|_{G}^{-(s-1)}$ as long as $s<Q / 2$.

Finally, it is worth mentioning that a byproduct of our elementary approach concerns the symbol properties of the Carnot-Caratheodory norm (or of any equivalent gauge). For general hypoelliptic families of vector fields, the norm is not always a symbol of order $\mathbf{1}$ (see section §3). On the contrary, on Carnot groups, it happens to always be equivalent to such a symbol (Proposition 7). At the end of the article, we discuss sufficient conditions for this property to hold for families of hypoelliptic vector fields, based either on the order $m$ of the Hörmander condition (Theorem 14), or on the way the commutators are structured (Theorems 15 and 16).

The structure of the article goes as follows. Section 1 is a brief survey of calculus on Carnot groups. It also sets the notations used subsequently. Section 2 contains the actual proof of theorem 1 and concludes on theorem 13, which is the homogeneous variant of the previous statement. Section 3 adresses an open question regarding families of vector fields that satisfy a Hörmander bracket condition, but lack an underlying group structure.

I would like to express my gratitude to J.-Y. Chemin, who brought this problem to my attention a long time ago, and to wish him a happy 60th birthday.

\section{A brief survey of calculus on Carnot Groups}

Let us first recall some classic definitions and facts about nilpotent Lie groups. We also introduce notations that will be needed in $\S 2$. For a more in-depth coverage of Lie groups, sub-Riemannian geometry and nilpotent groups, see e.g. [22], [13], [26] or the introduction of [3].

\subsection{Left-invariant vector fields and the exponential map}

Let us consider a Lie group $G$ and $\mathfrak{g}=T_{e} G$ its Lie algebra; $e$ denotes the unit element of $G$. Lefttranslation is defined by $L_{g}(h)=g h$.

Definition - A vector field $\xi$ is called left-invariant if $\left(L_{g}\right)_{*} \circ \xi=\xi \circ L_{g}$. Such a vector field is entirely determined by $v=\xi(e) \in \mathfrak{g}$. To signify that $v$ generates $\xi$, one writes $\xi=v^{L}$ thus:

$$
v^{L}(g)=d\left(L_{g}\right)_{\mid e}(v) .
$$

The tangent bundle $T G$ identifies to $G \times \mathfrak{g}$ by the map $(g, v) \mapsto\left(g, v^{L}(g)\right)$.

The flow $\Phi_{t}^{v}$ of a left-invariant vector field $v^{L}$ exists for all time. Indeed, one has $\Phi_{t}^{v}(g)=L_{g} \circ \Phi_{t}^{v}(e)$, which implies that $\Phi_{t+s}^{v}(e)=L_{\Phi_{s}^{v}(e)} \circ \Phi_{t}^{v}(e)$, thus allowing the flow to be extended globally once it has been constructed locally.

Definition - The exponential map exp $: \mathfrak{g} \rightarrow G$ is defined by $\exp (v)=\Phi_{1}^{v}(e)$ where $\left(\Phi_{t}^{v}\right)_{t \in \mathbb{R}}$ is the flow of the left-invariant vector field $v^{L}$.

One can check that the flow of $v^{L}$ starting from $g \in G$ is $\Phi_{t}^{v}(g)=g \exp (t v)$. In particular,

$$
\exp (s w) \exp (t v)=\Phi_{t}^{v}(\exp (s w)) \quad \text { and } \quad d \exp _{\mid 0}=\operatorname{Id}_{\mathfrak{g}} .
$$

\subsection{The Baker-Campbell-Hausdorff formula}

The commutator of two left-invariant vector fields is also a left-invariant field. Therefore, the commutator of $u, v \in \mathfrak{g}$ is defined by $[u, v]=\left[u^{L}, v^{L}\right](e) \in \mathfrak{g}$. The product law of $G$ induces an extremely rigid relation between exponentials, known as the Baker-Campbell-Hausdorff formula:

$$
\exp (u) \exp (v)=\exp (\mu(u, v))
$$


where $\mu(u, v)=u+v+\frac{1}{2}[u, v]+\frac{1}{12}([u,[u, v]]+[v,[v, u]])+\ldots$ is a universal Lie serie in $u, v$ i.e. an expression consisting of the iterated commutators of $u$ and $v$. In general, this formula holds provided $u$ and $v$ are small enough for the series to converge (see e.g. $[26, \S 1.3]$ ). Subsequently, one will only use the linear part of (9) with respect to one variable:

$$
d \mu(u, \cdot)_{\mid 0}(w)=\frac{\operatorname{ad}(u)}{1-e^{-\operatorname{ad}(u)}}(w)=w+\sum_{n=1}^{\infty} \frac{(-1)^{n} B_{n}}{n !} \underset{n \text { times } u}{[u, \ldots,[u, w]]}
$$

where $B_{n}$ are the Bernoulli numbers (i.e. $\left.\frac{x}{e^{x}-1}=\sum \frac{B_{n}}{n !} x^{n}\right)$ and $\operatorname{ad}(u)=[u, \cdot]$. This formula is classical and can be found e.g. in [26] or [19].

\subsection{Stratification}

From now on, $G$ is supposed to be stratified, i.e. it is a Carnot group as defined in the introduction of this paper. A stratified group is, in particular, nilpotent of step $m$. Moreover, elementary linear algebra gives restrictions on the possible dimensions $q_{j}=\operatorname{dim} V_{j}$ of the strata:

$$
q_{2} \leq \frac{q_{1}\left(q_{1}-1\right)}{2} \quad \text { and for } j \geq 2, \quad q_{j+1}<q_{1} q_{j}
$$

The last inequality is strict because of the Jacobi identity $[u,[v, w]]-[v,[u, w]]=-[w,[u, v]]$. For an exact count of the possible relations, see e.g. [25].

Proposition 2 Let $G$ be a Carnot group. Then exp $: \mathfrak{g} \rightarrow G$ is a global diffeomorphism that allows $G$ to be identified with the set $\mathfrak{g}$ equipped with the group law $u * v=\mu(u, v)$. The identity element is 0 and the inverse of $u$ is $-u$.

Proof. This claim is very standard so one only sketches the proof briefly. As $d \exp _{\mid 0}=\operatorname{Id}_{\mathfrak{g}}$, there exists a neighborhood $U_{0}$ of $e$ in $G$ and $V_{0}$ of 0 in $\mathfrak{g}$ such that exp : $V_{0} \rightarrow U_{0}$ is a diffeomorphism. As $G$ is connected, it is generated by any neighborhood of $e$ and in particular by $U_{0}=\exp \left(V_{0}\right)$. But, as $\mathfrak{g}$ is nilpotent, the expression $\mu(u, v)$ is a Lie polynomial of order $m$, thus (9) holds for any $u, v \in \mathfrak{g}$. Combining these facts implies that the exponential map is surjective. Next, one can show that the pair ( $\mathfrak{g}$, exp) is a covering space of $G$. Indeed, given $g=\exp (v) \in G$, one gets a commutative diagram of diffeomorphisms:

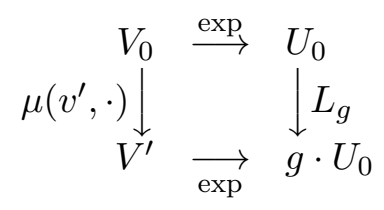

for each $v^{\prime} \in \mathfrak{g}$ such that $\exp \left(v^{\prime}\right)=g$. Finally, by a standard covering space argument based on the fact that $\mathfrak{g}$ is path connected (as vector space) and $G$ is simply connected (in the stratification assumption), one can claim that the exponential map is a global diffeomorphism.

Example - The Heisenberg group $\mathbb{H}$ can be realized as a set of upper-triangular matrices with diagonal entries equal to 1 . The group law in $\mathbb{H}$ is the multiplication of matrices:

$$
\left(\begin{array}{ccc}
1 & p & r \\
0 & 1 & q \\
0 & 0 & 1
\end{array}\right) \cdot\left(\begin{array}{ccc}
1 & p^{\prime} & r^{\prime} \\
0 & 1 & q^{\prime} \\
0 & 0 & 1
\end{array}\right)=\left(\begin{array}{ccc}
1 & p+p^{\prime} & r+r^{\prime}+p q^{\prime} \\
0 & 1 & q+q^{\prime} \\
0 & 0 & 1
\end{array}\right)
$$

The Lie algebra of $\mathbb{H}$ is

$$
\mathfrak{h}=\left\{\left(\begin{array}{ccc}
0 & p & r \\
0 & 0 & q \\
0 & 0 & 0
\end{array}\right)=p Y_{1}+q Y_{2}+r Y_{3} ; p, q, r \in \mathbb{R}\right\} .
$$


Left-invariant vector fields on $\mathbb{H}$ are linear combinations of

$$
Y_{1}^{L}(g)=Y_{1}, \quad Y_{2}^{L}(g)=Y_{2}+p Y_{3} \quad \text { and } \quad Y_{3}^{L}(g)=Y_{3} \quad \text { where } \quad g=\left(\begin{array}{ccc}
1 & p & r \\
0 & 1 & q \\
0 & 0 & 1
\end{array}\right) .
$$

$H$ is a stratified nilpotent group with $V_{1}=\operatorname{Span}\left(Y_{1}, Y_{2}\right)$ and $V_{2}=\operatorname{Span}\left(Y_{3}\right)$. The exponential map is the usual exponential of nilpotent matrices. It transfers the group structure to $\mathfrak{h} \simeq \mathbb{R}^{3}$ by (9):

$$
\begin{gathered}
(p, q, r)_{\mathfrak{h}} \simeq\left(\begin{array}{ccc}
0 & p & r \\
0 & 0 & q \\
0 & 0 & 0
\end{array}\right), \quad \exp \left(\begin{array}{ccc}
0 & p & r \\
0 & 0 & q \\
0 & 0 & 0
\end{array}\right)=\left(\begin{array}{ccc}
1 & p & r+\frac{1}{2} p q \\
0 & 1 & q \\
0 & 0 & 1
\end{array}\right), \\
\mu\left((p, q, r)_{\mathfrak{h}},\left(p^{\prime}, q^{\prime}, r^{\prime}\right)_{\mathfrak{h}}\right)=\left(p+p^{\prime}, q+q^{\prime}, r+r^{\prime}+\frac{1}{2}\left(p q^{\prime}-q p^{\prime}\right)\right)_{\mathfrak{h}} .
\end{gathered}
$$

In exponential coordinates on $\mathfrak{h} \simeq \mathbb{R}^{3}$, the left-invariant vector fields thus take the following form:

$$
\tilde{Y}_{1}^{L}(p, q, r)=\partial_{p}-\frac{1}{2} q \partial_{r}, \quad \tilde{Y}_{2}^{L}(p, q, r)=\partial_{q}+\frac{1}{2} p \partial_{r}, \quad \tilde{Y}_{3}^{L}(p, q, r)=\partial_{r} .
$$

The two different expressions of the fields correspond to the change of variables $(p, q, r) \mapsto\left(p, q, r-\frac{1}{2} p q\right)$.

\section{Remarks}

- In general (even if $G$ is connected and nilpotent), only the Lie group action can be recovered from the exponential map but not $G$ itself. For example, $G=\{z \in \mathbb{C} ;|z|=1\} \simeq \mathbb{S}^{1}$ with rotation law $\left(z, z^{\prime}\right) \mapsto z z^{\prime}$ is a nilpotent group. One has $\mathfrak{g}=\mathbb{R}$ and $\mu(x, y)=x+y$ but the exponential map is $\exp (x)=e^{i x}$ and is obviously not a global diffeomorphism.

- Combined with (10), the commutative diagram of the proof of Proposition 2 provides a general formula for the differential of the exponential map, which we will need later on. For $v, w \in \mathfrak{g}$ and $g=\exp (v)$, one has:

$$
\left.d \exp _{\mid v}(w)=\sum_{n=0}^{m-1} \frac{(-1)^{n}}{(n+1) !} \underset{n \text { times } v}{[v, \ldots]}\right]^{L}(g) .
$$

For example, on the Heisenberg group, one gets $d \exp _{\mid v}(w)=\left(w-\frac{1}{2}[v, w]\right)^{L}(g)$.

Proposition 3 For any indices $j, k \in\{1, \ldots, m\}$, one has

$$
\left[V_{j}, V_{k}\right] \subset \begin{cases}V_{j+k} & \text { if } j+k \leq m \\ \{0\} & \text { otherwise }\end{cases}
$$

Proof. By convention, let us write $V_{n}=\{0\}$ if $n>m$. For $k=1$, the property holds by definition. For $k=2$, as $V_{2}=\left[V_{1}, V_{1}\right]$, any element can be written $[X, Y]$ with $X, Y \in V_{1}$. For $Z \in V_{j}$, one uses the identity $[A, B C]=[A, B] C+B[A, C]$ to get:

$$
[Z,[X, Y]]=[[Z, X], Y]-[[Z, Y], X] \in\left[\left[V_{j}, V_{1}\right], V_{1}\right] \subset\left[V_{j+1}, V_{1}\right] \subset V_{j+2} .
$$

Next, one proceeds recursively. Assuming that for some $k \geq 2$, one has $\left[V_{j}, V_{k}\right] \subset V_{j+k}$ for any $j$, then given $Z \in V_{k+1}=\left[V_{1}, V_{k}\right]$, one writes $Z=[X, \zeta]$ with $X \in V_{1}$ and $\zeta \in V_{k}$. Then for any $W \in V_{j}$, the Jacobi identity gives

$$
[W, Z]=[W,[X, \zeta]]=-[X,[\zeta, W]]-[\zeta,[W, X]] \in\left[V_{1}, V_{j+k}\right]+\left[V_{k}, V_{j+1}\right] \subset V_{j+k+1}
$$

which makes the property hereditary in $k$. 


\subsection{Stratified dilations}

The next essential object in a Carnot group is the dilation of the Lie algebra:

$$
\forall r>0, \quad \delta_{r}=\sum_{j=1}^{m} r^{j} \pi_{j}
$$

where $\pi_{j}: \mathfrak{g} \rightarrow V_{j}$ is the projection onto $V_{j}$ with kernel $\bigoplus_{k \neq j} V_{k}$. Identifying $G$ to $\exp (\mathfrak{g})$, one gets a one parameter family of group automorphisms that we will simply denote by

$$
r g=\exp \circ \delta_{r} \circ \exp ^{-1}(g)
$$

for any $r>0$ and $g \in G$.

The next result is an immediate consequence of the definition but should later be compared with the scaling property (41) of the radial vector field.

Proposition 4 The dilation of a left-invariant vector field $v^{L}$ is given by:

$$
\left(\delta_{r} v\right)^{L}(r g)=\left(\left(L_{r g}\right)_{*} \circ \delta_{r} \circ\left(L_{g}\right)_{*}^{-1}\right)\left(v^{L}(g)\right) .
$$

Up to a constant factor, the Haar measure on $G$ is given by the Lebesgue measure on $\mathfrak{g} \simeq \mathbb{R}^{q}$ and is commonly denoted $d g$.

Proposition 5 One has:

$$
\forall \phi \in L^{1}(G), \quad \forall r>0, \quad \int_{G} \phi(r g) d g=r^{-Q} \int_{G} \phi(g) d g
$$

where $Q$ is the homogeneous dimension (5) of $G$. Note that when $m \neq 1$, one has $Q>q$.

Given $\left(\operatorname{arbitrary}^{1}\right)$ Euclidian norms $\|\cdot\|_{V_{j}}$ on each $V_{j}$ and $w=2 \operatorname{LCM}(1, \ldots, m)$, the anisotropic gauge-norm of either $v \in \mathfrak{g}$ or of $g=\exp (v) \in G$ is defined by:

$$
\|v\|_{\mathfrak{g}}=\|g\|_{G}=\left(\sum_{j=1}^{m}\left\|\pi_{j}(v)\right\|_{V_{j}}^{w / j}\right)^{1 / w} .
$$

The gauge norm is homogeneous in the following sense:

$$
\|r g\|_{G}=r\|g\|_{G} \quad \text { and } \quad\left|\square_{g, r}\right|=c_{0} r^{Q}
$$

with a uniform constant $c_{0}$ and where the gauge-ball is defined by

$$
\square_{g, r}=\left\{h \in G ;\left\|h^{-1} g\right\|_{G}<r\right\} .
$$

Remark - The intrinsic metric objects of $G$ are the so-called Carnot balls defined as the set of points that can be connected to a center $g_{0} \in G$ by an absolutely continuous path $\gamma$ whose velocity is subunitary for almost every time i.e. such that $\dot{\gamma}(t) \in\left(L_{\gamma(t)}\right)_{*}\left(B_{0}\right)$ where $B_{0} \subset V_{1}$ is a fixed Euclidian ball of the first layer of the stratification (up to some choice of a Euclidian metric on $V_{1}$ ). However, the ball-box theorem [22] states that such intrinsic objects can be sandwiched between two gauge-balls of comparable radii. For our purpose (the analysis of Sobolev spaces), one can thus deal only with gauge-balls without impeding the generality.

\footnotetext{
${ }^{1}$ For a given basis of $\mathfrak{g}$ adapted to the stratification, one will chose here a Euclidian structure that renders this basis orthonormal. This is the natural choice when one proceeds to the identification $\mathfrak{g} \simeq \mathbb{R}^{q}$ through this basis.
} 


\subsection{Horizontal derivatives and Sobolev spaces on $G$}

Vector fields $\xi$ on $G$ are identified with derivation operators on $C^{\infty}(G)$ by the Lie derivative formula:

$$
(\xi \phi)(g)=d \phi_{\mid g}(\xi(g)) .
$$

Definition - The horizontal derivatives are the left-invariant vector-fields associated with $V_{1}$.

Let us consider a basis $\left(Y_{\ell}\right)_{1 \leq \ell \leq q}$ of $\mathfrak{g}$ that is adapted to the stratification, i.e. :

$$
V_{j}=\operatorname{Span}\left(Y_{\ell}\right)_{n_{j-1}<\ell \leq n_{j}}
$$

where $n_{j}$ is defined by (3). An horizontal derivative is thus a vector field

$$
\xi=\sum_{j \leq n_{1}} \alpha_{j} Y_{j}^{L}=\alpha \cdot \nabla_{G}
$$

where $\alpha=\left(\alpha_{1}, \ldots, \alpha_{n_{1}}\right) \in \mathbb{R}^{n_{1}}$ and $\nabla_{G}$ is defined by (4). Non-commutative multi-indices are defined as follows: for $\gamma=\left(\gamma_{1}, \ldots, \gamma_{\ell}\right) \in\left\{1, \ldots, n_{1}\right\}^{\ell}$, one writes $l=|\gamma|$ and $\nabla_{G}^{\gamma}=Y_{\gamma_{1}}^{L} \circ \cdots \circ Y_{\gamma_{l}}^{L}$.

Let us unfold the commutator structure in $\mathfrak{g}$ with the following notation:

$$
\left[Y_{\ell_{1}}, \ldots,\left[Y_{\ell_{n}}, Y_{\ell_{n+1}}\right]\right]=\sum_{\ell^{\prime}} \kappa\left(\ell_{1}, \ldots, \ell_{n}, \ell_{n+1} ; \ell^{\prime}\right) Y_{\ell^{\prime}}
$$

Note that according to (12), one can warrant that $\kappa\left(\ell_{1}, \ldots, \ell_{n}, \ell_{n+1} ; \ell^{\prime}\right)=0$ if $\omega_{\ell^{\prime}} \neq \omega_{\ell_{1}}+\ldots+\omega_{\ell_{n+1}}$.

Remark - To simplify computations, one can always assume that the basis is chosen such that:

$$
\forall \ell \in\{1, \ldots, q\}, \quad Y_{\ell}=\left[Y_{\alpha_{1}(\ell)}, \ldots,\left[Y_{\alpha_{k-1}(\ell)}, Y_{\alpha_{k}(\ell)}\right]\right]
$$

where $k=\omega_{\ell}$ and $\alpha_{i}(\ell) \leq n_{1}$. Indeed, the Lie algebra is linearly generated by the commutators of the restricted family $\nabla_{G}$ and one just has to extract a basis from it.

Example - On the Heisenberg group $\mathbb{H}$, the horizontal derivatives are left-invariant vector fields of the form $\xi=\left(\alpha Y_{1}+\beta Y_{2}\right)^{L}$ for $\alpha, \beta \in \mathbb{R}$.

Definition - For $s \in \mathbb{N}$, the Sobolev space $H^{s}(G)$ consists of the functions such that each composition of at most $s$ horizontal derivatives belongs to $L^{2}(G)$. The norm is defined (up to the choice of the $Y_{\ell}$ ) by:

$$
\|\phi\|_{H^{s}(G)}^{2}=\sum_{|\gamma| \leq s} \int_{G}\left|\nabla_{G}^{\gamma} \phi(g)\right|^{2} d g
$$

with $\gamma$ a non-commutative multi-index.

Remark - The space $H^{2}(G)$ is the domain of the hypo-elliptic Laplace operator

$$
\mathcal{L}_{G}=-\sum_{\ell \leq n_{1}}\left(Y_{\ell}^{L}\right)^{*} Y_{\ell}^{L}
$$

A celebrated result of L. Hörmander [18] states that $H^{s}(G) \subset H_{\mathrm{loc}}^{s / m}\left(\mathbb{R}^{q}\right)$ where the last Sobolev space is the classical one (homogeneous and isotropic) on $\mathbb{R}^{q}$. 


\subsection{Exponential coordinates on a stratified group}

Given a basis $\left(Y_{\ell}\right)_{1 \leq \ell \leq q}$ of $\mathfrak{g}$ adapted to the stratification, one can define a natural coordinate system on $G$, called exponential coordinates. Given $g=\exp (v) \in G$, its coordinates $x(g)=\left(x_{\ell}(g)\right)_{1 \leq \ell \leq q} \in \mathbb{R}^{q}$ are defined by:

$$
v=\sum_{\ell=1}^{q} x_{\ell}(g) Y_{\ell}
$$

The projections $\left(\pi_{j}\right)_{1 \leq j \leq m}$ introduced in (13) are:

$$
\forall j \in\{1, \ldots, m\}, \quad \pi_{j}(v)=\sum_{\ell=1+n_{j-1}}^{n_{j}} x_{\ell}(g) Y_{\ell} .
$$

In exponential coordinates, the expression of stratified dilations (14) is:

$$
\forall \ell \in\{1, \ldots, q\}, \quad x_{\ell}(r g)=r^{\omega_{\ell}} x_{\ell}(g) .
$$

The gauge norm (17) is given (for some fixed large $w \in \mathbb{N}$ ) by:

$$
\|g\|_{G}=\|x(g)\|_{\mathfrak{g}}=\left(\sum_{j=1}^{m}\left(\sum_{\ell=1+n_{j-1}}^{n_{j}}\left|x_{\ell}(g)\right|^{2}\right)^{w / j}\right)^{1 /(2 w)} .
$$

One could however take any uniformly equivalent quantity as a gauge norm, which will be the case subsequently, after proposition 7 .

Example - With the previous notations, the exponential coordinates on the Heisenberg group $\mathbb{H}$ are:

$$
x_{1}(g)=p, \quad x_{2}(g)=q, \quad x_{3}(g)=r-\frac{1}{2} p q \quad \text { for } \quad g=\left(\begin{array}{ccc}
1 & p & r \\
0 & 1 & q \\
0 & 0 & 1
\end{array}\right) \in \mathbb{H} .
$$

\subsection{Left-invariant basis of vector fields}

When doing explicit computations, it is natural to identify $\mathfrak{g}$ with $\mathbb{R}^{q}$ through the previous coordinates. Given $v \in \mathfrak{g}$, the left-invariant vector field $v^{L}$ on $G$ defined by (8) matches a corresponding vector field on $\mathfrak{g} \simeq \mathbb{R}^{q}$ that we will denote by $\tilde{v}^{L}$. According to the Baker-Campbell-Hausdorff formula (10):

$$
\forall x=\left(x_{1}, \ldots, x_{q}\right) \in \mathfrak{g}, \quad \tilde{v}^{L}(x)=v+\sum_{n=1}^{m-1} \sum_{\ell_{1}, \ldots, \ell_{n}} \frac{(-1)^{n} B_{n}}{n !} x_{\ell_{1}} \ldots x_{\ell_{n}}\left[Y_{\ell_{1}}, \ldots,\left[Y_{\ell_{n}}, v\right]\right]
$$

where each of the $\ell_{i}$ ranges over $\{1, \ldots, q\}$.

After the identification $\mathfrak{g} \simeq \mathbb{R}^{q}$ and to avoid confusion, let us denote $Y_{\ell}$ by $\partial_{\ell}$ : the vectors $\left(\partial_{\ell}\right)_{1 \leq \ell \leq q}$ are the dual basis of the coordinates $\left(x_{\ell}\right)_{1 \leq \ell \leq q}$. The left-invariant basis then becomes explicit:

$$
\forall x=\left(x_{1}, \ldots, x_{q}\right) \in \mathfrak{g}, \quad \tilde{Y}_{\ell}^{L}(x)=\partial_{\ell}+\sum_{\ell^{\prime}=1}^{q} \zeta_{\ell, \ell^{\prime}}\left(x_{1}, \ldots, x_{q}\right) \partial_{\ell^{\prime}}
$$

with, thanks to (29) and (21):

$$
\zeta_{\ell, \ell^{\prime}}(x)=\sum_{n=1}^{m-1} \frac{(-1)^{n} B_{n}}{n !} \sum_{\ell_{1}, \ldots, \ell_{n}} \kappa\left(\ell_{1}, \ldots, \ell_{n}, \ell ; \ell^{\prime}\right) x_{\ell_{1}} \ldots x_{\ell_{n}} .
$$


Let us point out that $\zeta_{\ell, \ell^{\prime}}=0$ if $\omega_{\ell^{\prime}} \leq \omega_{\ell}$ (because $\kappa$ vanishes), thus the left-invariant correction to $\partial_{\ell}$ only involves derivatives of a strictly higher weight. In other words, the indices in (30) can be restricted to $\ell^{\prime}>n_{\omega_{\ell}}=q_{1}+q_{2}+\ldots+q_{\omega_{\ell}}$.

Note also that $\zeta \in C^{\infty}\left(\mathbb{R}^{q}, \mathcal{M}_{q, q}(\mathbb{R})\right)$ and $\zeta(0)=0$. More precisely, this matrix represents the differential action of left-translations, expressed in exponential coordinates:

$$
\left(d L_{g}\right)_{\mid e} \equiv \operatorname{Id}_{\mathbb{R}^{q}}+\zeta(x(g)) .
$$

Moreover, as $\left|x_{\ell}(g)\right| \lesssim\|g\|_{G}^{\omega_{\ell}}$, one has $\left|\zeta_{\ell, \ell^{\prime}}(x(g))\right| \lesssim\|g\|_{G}^{\omega_{\ell^{\prime}}-\omega_{\ell}}$.

\section{Proof of Theorem 1}

This section is devoted to the proof of the main statement. The key idea is to prove the result for $s=1$ and then "push" the result up to the maximal regularity using only integrations by part. Adding an interpolation step once the result is known for $s=1$, but before pushing it to a higher regularity, allows one to capture all eligible fractional derivatives. The actual proof is written in the last subsection $\S 2.5$ but some preliminary results are required.

\subsection{Symbol classes $S_{n}^{\alpha}(G)$}

Symbol classes are a convenient way to classify the coefficients involved in the computations in terms of how they vanish at the origin.

Definition - For $\alpha \in \mathbb{R}_{+}$and $n \in \mathbb{N} \cup\{\infty\}$, the symbol class $S_{n}^{\alpha}(G)$ is defined as the set of functions $\phi \in L_{\mathrm{loc}}^{\infty}(G)$ such that for any multi-index $\gamma$ of length $|\gamma| \leq n$, there exists a constant $C_{\gamma}>0$ that ensures the following inequality:

$$
\forall g \in G, \quad\|g\|_{G} \leq 1 \quad \Longrightarrow \quad\left|\nabla_{G}^{\gamma} \phi(g)\right| \leq C_{\gamma}\|g\|_{G}^{(\alpha-|\gamma|)_{+}} .
$$

For example, the symbols of class $S_{0}^{0}(G)=L_{\text {loc }}^{\infty}(G)$ are only required to be bounded near the origin. The symbol class $S_{\infty}^{\alpha}(G)$ is also denoted $S^{\alpha}(G)$.

The following properties hold.

1. The Leibnitz formula gives

$$
\phi \in S_{m}^{\alpha}(G), \quad \psi \in S_{n}^{\beta}(G) \quad \Longrightarrow \quad \phi \psi \in S_{\min (m, n)}^{\alpha+\beta}(G) .
$$

2. As $Y_{\ell}^{L}$ is a linear combination of derivatives $\nabla_{G}^{\gamma}$ of length $|\gamma|=\omega_{\ell}$, one has (if $n \geq \omega_{\ell}$ ):

$$
\phi \in S_{n}^{\alpha}(G) \quad \Longrightarrow \quad Y_{\ell}^{L} \phi \in S_{n-\omega_{\ell}}^{\left(\alpha-\omega_{\ell}\right)_{+}}(G)
$$

3. As smooth functions are locally bounded, one has also: $S_{\alpha-1}^{\alpha}(G) \cap C^{\infty}(G) \subset S^{\alpha}(G)$.

The coordinates and the coefficients of the left-invariant vector fields belong to the following classes.

Proposition 6 One has

$$
\forall \ell \in\{1, \ldots, q\}, \quad x_{\ell}(g) \in S^{\omega_{\ell}}(G)
$$

and

$$
\forall \ell, \ell^{\prime} \in\{1, \ldots, q\}, \quad \zeta_{\ell, \ell^{\prime}}\left(x_{1}(g), \ldots, x_{q}(g)\right) \in S^{\omega_{\ell^{\prime}}-\omega_{\ell}}(G) .
$$


Proof. We already observed that $\left|x_{\ell}(g)\right| \lesssim\|g\|_{G}^{\omega_{\ell}}$ thus $x_{\ell}(g) \in S_{0}^{\omega_{\ell}}(G)$. Next, using (30), one gets:

$$
Y_{\ell_{0}}^{L}\left(x_{\ell}(g)\right)=\delta_{\ell_{0}, \ell}+\zeta_{\ell_{0}, \ell}(x(g))= \begin{cases}\zeta_{\ell_{0}, \ell}(x(g)) & \text { if } \omega_{\ell_{0}}<\omega_{\ell}, \\ \delta_{\ell_{0}, \ell} & \text { if } \omega_{\ell_{0}}=\omega_{\ell} \\ 0 & \text { if } \omega_{\ell_{0}}>\omega_{\ell} .\end{cases}
$$

Assuming $\ell_{0} \in\left\{1, \ldots, n_{1}\right\}$, one gets $\left|\nabla_{G} x_{\ell}(g)\right| \leq C\|g\|_{G}^{\omega_{\ell}-1}$ thus $x_{\ell}(g) \in S_{1}^{\omega_{\ell}}(G)$. One can now bootstrap this partial result in the expression (31), which gets us $\zeta_{\ell, \ell^{\prime}}(x(g)) \in S_{1}^{\omega_{\ell^{\prime}}-\omega_{\ell}}(G)$. The previous expression now reads $\nabla_{G} x_{\ell}(g) \in S_{1}^{\omega_{\ell}-1}$ and thus $x_{\ell}(g) \in S_{2}^{\omega_{\ell}}(G)$. Iterating this process leads to $x_{\ell}(g) \in S_{\infty}^{\omega_{\ell}}(G)$ and $\zeta_{\ell, \ell^{\prime}}(x(g)) \in S_{\infty}^{\omega_{\ell^{\prime}}-\omega_{\ell}}(G)$.

The key result is that one can adjust the gauge norm to be a symbol of order 1 (see also $\S 3$ ).

Proposition 7 There exists a symbol $\rho(g) \in S_{1}^{1}(G)$ that is uniformly equivalent to the gauge norm. For higher-order derivatives, it satisfies for any multi-index $\gamma$ :

$$
\forall g \in G, \quad \rho(g) \leq 1 \quad \Longrightarrow \quad\left|\nabla_{G}^{\gamma} \rho\right| \leq \frac{C_{\gamma}}{\rho^{|\gamma|-1}} .
$$

Moreover, there exists $w \in \mathbb{N}$ such that $\rho^{w} \in S^{w}(G)$.

Proof. Let us now modify the gauge norm (28) into the uniformly equivalent gauge:

$$
\rho(g)=\left(\sum_{\ell=1}^{q}\left|x_{\ell}(g)\right|^{w / w_{\ell}}\right)^{1 / w}
$$

with $w=2 \operatorname{LCM}(1, \ldots, m)$ to ensure that each $w / \omega_{\ell} \in 2 \mathbb{N}$. In particular, $\rho(g)^{w} \in C^{\infty}(G)$. Next, one computes the first horizontal derivative of the norm, using (30):

$$
\nabla_{G} \rho=\frac{\nabla_{G}\left(\rho^{w}\right)}{w \rho^{w-1}}=\left(\frac{1}{\rho^{w-1}}\left[x_{\ell}^{w-1}+\sum_{\ell^{\prime}=1}^{q} \frac{\zeta_{\ell, \ell^{\prime}}(x) x_{\ell^{\prime^{\prime}}}^{\frac{w}{\omega_{\ell^{\prime}}}-1}}{\omega_{\ell^{\prime}}}\right]\right)_{\ell=1 \ldots n_{1}} .
$$

The expression in square brackets is a symbol of class $S^{w-1}(G)$ because of (34) and (35) and $\omega_{\ell}=1$ for $\ell \leq n_{1}$. Thus $\nabla_{G} \rho$ is bounded near the origin which means that the modified gauge $\rho$ belongs to $S_{1}^{1}(G)$. Next, one observes that for any $\alpha \geq 1$, if $\theta \in S^{\alpha}(G)$ then

$$
\nabla_{G}\left(\frac{\theta}{\rho^{\alpha}}\right)=\left(\frac{\nabla_{G} \theta}{\rho^{\alpha-1}}-\alpha \frac{\theta}{\rho^{\alpha}} \nabla_{G} \rho\right) \frac{1}{\rho}=\left(\frac{\theta_{1}}{\rho^{\alpha-1}}+\frac{\theta_{2}}{\rho^{\alpha+w-1}}\right) \frac{1}{\rho}
$$

with $\theta_{1} \in S^{\alpha-1}(G)$ and $\theta_{2} \in S^{\alpha+w-1}(G)$. One can thus claim by recurrence on the length of the multi-index $\gamma$ that

$$
\nabla_{G}^{\gamma} \rho=\left(\theta_{\gamma, 0}+\sum_{k} \frac{\theta_{\gamma, k}}{\rho^{\alpha_{k}}}\right) \frac{1}{\rho^{|\gamma|-1}}
$$

where $\theta_{\gamma, k} \in S^{\alpha_{k}}(G)$ is a polynomial in $x_{\ell}(g)$ with $\alpha_{k} \geq 1$ and $\theta_{\gamma, 0}$ is a polynomial. Note that a polynomial in $S^{0}(G)$ is necessarily the sum of a constant and a polynomial in $S^{1}(G)$ and that, by (30), the horizontal derivatives of a polynomial are also a polynomial. This ensures (36). The final assertion about $\|\cdot\|_{G}^{w}$ follows immediately from (34).

From now one, one will modify the gauge norm accordingly and assume that $\|\cdot\|_{G}=\rho \in S_{1}^{1}(G)$. 


\subsection{The radial vector field}

The infinitesimal generator of dilations on $\mathfrak{g}$ is the linear operator $\tilde{R}: \mathfrak{g} \rightarrow \mathfrak{g}$ defined by

$$
\tilde{R}=\sum_{j=1}^{m} j \pi_{j}
$$

It is diagonalizable with positive eigenvalues; its trace $\operatorname{Tr} \tilde{R}=Q$ is the homogeneous dimension. One checks immediately that $\delta_{r}=e^{(\log r) R}$ thus $\tilde{R}(x)=\left.\frac{d}{d r} \delta_{r}(x)\right|_{r=1}$. The pair $(x, \tilde{R}(x))$ is a vector field on $\mathfrak{g}$ whose expression in exponential coordinates follows from $(26)$ :

$$
\forall x=\left(x_{1}, \ldots, x_{q}\right) \in \mathfrak{g}, \quad \tilde{R}(x)=\sum_{\ell=1}^{q} \omega_{\ell} x_{\ell} \partial_{\ell} .
$$

Its exponential lift is called the radial field on $G$ :

$$
R(g)=d \exp _{\mid v}(\tilde{R}(v))=\left.\frac{d}{d r}(r g)\right|_{r=1} .
$$

Proposition 8 The radial vector field is scaling invariant:

$$
R(r g)=\left(\left(L_{r g}\right)_{*} \circ \delta_{r} \circ\left(L_{g}\right)_{*}^{-1}\right)(R(g)) .
$$

Moreover, it can be expressed in terms of left-invariant derivatives:

$$
R(g)=\sum_{\ell=1}^{q} \sigma_{\ell}\left(x_{1}(g), \ldots, x_{q}(g)\right) Y_{\ell}^{L}(g)
$$

with $\sigma_{\ell}(x(g))=\omega_{\ell} x_{\ell}(g)+\sum_{n=1}^{m-1} \frac{(-1)^{n}}{(n+1) !} \sum_{\ell_{1}, \ldots, \ell_{n+1}} x_{\ell_{1}}(g) \cdots x_{\ell_{n+1}}(g) \cdot \omega_{\ell_{n+1}} \kappa\left(\ell_{1}, \ldots, \ell_{n+1} ; \ell\right) \in S^{\omega_{\ell}}(G)$.

\section{Remarks}

- Note that the variable $x_{\ell_{i}}$ that appears in the second term defining $\sigma_{\ell}$ must satisfy

$$
\omega_{\ell}=\omega_{\ell_{1}}+\ldots+\omega_{\ell_{n+1}}
$$

because if it is not the case, then $\kappa\left(\ell_{1}, \ldots, \ell_{n+1} ; \ell\right)=0$. In particular, as there are at least $n+1 \geq 2$ factors, one has $\omega_{\ell_{i}}<\omega_{\ell}$ for each $i$.

- Both expressions for $\tilde{R}=\sum \omega_{\ell} x_{\ell} \partial_{\ell}=\sum \sigma_{\ell} \tilde{Y}_{\ell}^{L}$ combined with (30) provide a remarkable identity embedded in the commutator structure. For any $\ell^{\prime} \in\{1, \ldots, q\}$ :

$$
\begin{gathered}
\sum_{n=1}^{m-1} \frac{(-1)^{n}}{(n+1) !} \sum_{\ell_{1}, \ldots, \ell_{n+1}} x_{\ell_{1}} \cdots x_{\ell_{n+1}} \omega_{\ell_{n+1}}\left(\kappa\left(\ell_{1}, \ldots, \ell_{n+1} ; \ell^{\prime}\right)+\sum_{\ell} \kappa\left(\ell_{1}, \ldots, \ell_{n+1} ; \ell\right) \zeta_{\ell, \ell^{\prime}}(x)\right) \\
=-\sum_{\ell} \omega_{\ell} x_{\ell} \zeta_{\ell, \ell^{\prime}}(x) .
\end{gathered}
$$

Note that when one substitutes $x=x(g)$, both sides are indeed symbols of class $S^{\omega_{\ell^{\prime}}}(G)$. 
Proof. Formula (41) follows e.g. from the identities $\tilde{R} \circ \delta_{r}=\delta_{r} \circ \tilde{R}$ and $\operatorname{ad} \circ \delta_{r}=\delta_{r} \circ$ ad $\circ \delta_{r}^{-1}$ :

$$
\begin{aligned}
R(r g) & =d \exp _{\mid \delta_{r}(v)} \circ \delta_{r} \circ \tilde{R}(v) \\
& =\left(L_{r g}\right)_{*} \circ\left(\frac{1-e^{-\operatorname{ad}\left(\delta_{r}(v)\right)}}{\operatorname{ad}\left(\delta_{r}(v)\right)}\right) \circ \delta_{r} \circ \tilde{R}(v) \\
& =\left(L_{r g}\right)_{*} \circ \delta_{r} \circ\left(\frac{1-e^{-\operatorname{ad}(v)}}{\operatorname{ad}(v)}\right) \circ \tilde{R}(v) \\
& =\left(\left(L_{r g}\right)_{*} \circ \delta_{r} \circ\left(L_{g}\right)_{*}^{-1}\right)(R(g)) .
\end{aligned}
$$

The definition of $R(g)$ with $g=\exp (v)$ also reads

$$
R(g)=\sum_{\ell=1}^{q} \omega_{\ell} x_{\ell}(g)\left(d \exp _{\mid v} Y_{\ell}\right)
$$

Combining the expression for the differential of exp given by (11), the identity $[u, v]^{L}=\left[u^{L}, v^{L}\right]$ and the fact that $v=\sum x_{\ell}(g) Y_{\ell}$ give:

$$
\begin{aligned}
R(g) & =\sum_{\ell=1}^{q} \omega_{\ell} x_{\ell}(g)\left(\sum _ { n = 0 } ^ { m - 1 } \frac { ( - 1 ) ^ { n } } { ( n + 1 ) ! } \left[\begin{array}{c}
\left.\left.v, \ldots,\left[v, Y_{\ell}\right]\right]\right)^{L}(g) \\
n \text { times } v
\end{array}\right.\right. \\
& =\sum_{\ell=1}^{q} \omega_{\ell} x_{\ell}(g)\left(Y_{\ell}^{L}(g)+\sum_{n=1}^{m-1} \sum_{\ell_{1}, \ldots, \ell_{n}} \frac{(-1)^{n}}{(n+1) !} x_{\ell_{1}}(g) \ldots x_{\ell_{n}}(g)\left[Y_{\ell_{1}}, \ldots,\left[Y_{\ell_{n}}, Y_{\ell}\right]\right]^{L}(g)\right) .
\end{aligned}
$$

This formula can be further simplified into (42) by using (21). The symbol property comes from (34) and the restriction on non-vanishing indices imposed by (21).

Examples - The previous computation can be simplified further by observing the anti-symmetrical role of $\ell_{n}$ and $\ell_{n+1}$ in $\omega_{\ell_{n+1}}\left[Y_{\ell_{n}}, Y_{\ell_{n+1}}\right]$ if $\omega_{\ell_{n}}=\omega_{\ell_{n+1}}$. For $m \leq 4$, one thus gets the following expressions for the radial field.

1. For a group of step $m=2$, the radial field is given by:

$$
R(g)=\sum_{\ell=1}^{q} \omega_{\ell} x_{\ell}(g) Y_{\ell}^{L}(g) .
$$

On the Heisenberg group $\mathbb{H}$ with exponential coordinates introduced in $\S 1.6$, this formula boils down, as expected, to the following one:

$$
R(g)=\left(p Y_{1}+q Y_{2}+2\left(r-\frac{1}{2} p q\right) Y_{3}\right)^{L}(g)=p \partial_{p}+q \partial_{q}+2 r \partial_{r} .
$$

2. For a group of step $m=3$, the radial field is "corrected" along $V_{3}$ :

$$
R(g)=\sum_{\ell=1}^{q} \omega_{\ell} x_{\ell}(g) Y_{\ell}^{L}-\frac{1}{2} \sum_{\substack{1 \leq \ell_{1} \leq n_{1} \\ n_{1}<\ell_{2} \leq n_{2}}} x_{\ell_{1}}(g) x_{\ell_{2}}(g)\left[Y_{\ell_{1}}, Y_{\ell_{2}}\right]^{L} .
$$

3. For step $m=4$, its expression involves a further "correction" along $V_{4}$ that is split among two types of commutators:

$$
\begin{aligned}
R(g)= & \sum_{\ell=1}^{q} \omega_{\ell} x_{\ell}(g) Y_{\ell}^{L}-\frac{1}{2} \sum_{\substack{1 \leq \ell_{1} \leq n_{1} \\
n_{1}<\ell_{2} \leq \mathbf{n}_{3}}} x_{\ell_{1}}(g) x_{\ell_{2}}(g)\left[Y_{\ell_{1}}, Y_{\ell_{2}}\right]^{L} \\
& +\frac{1}{6} \sum_{\substack{1 \leq \ell_{1}, \ell_{2} \leq n_{1} \\
n_{1}<\ell_{3} \leq n_{2}}} x_{\ell_{1}}(g) x_{\ell_{2}}(g) x_{\ell_{3}}(g)\left[Y_{\ell_{1}},\left[Y_{\ell_{2}}, Y_{\ell_{3}}\right]\right]^{L} .
\end{aligned}
$$


Proposition 9 The gauge norm (37) and the radial field are related by the following formula:

$$
\forall s>0, \quad \frac{1}{\|\cdot\|_{G}^{2 s}}=-\frac{1}{2 s} R\left(\frac{1}{\|\cdot\|_{G}^{2 s}}\right) .
$$

Proof. Applying the chain rule, one gets:

$$
\frac{\lambda(g)}{\|g\|_{G}^{2 s}}=-\frac{1}{2 s} R\left(\frac{1}{\|g\|_{G}^{2 s}}\right)
$$

with $\lambda(g)=\frac{R\left(\|g\|_{G}\right)}{\|g\|_{G}}$ and where the field $R$ is obviously computed at the same point $g \in G$ as the function that is being derivated. Let us also observe that:

$$
\lambda(g)=\frac{R\left(\|g\|_{G}^{w}\right)}{w\|g\|_{G}^{w}}
$$

for any $w \in \mathbb{N}^{*}$ and in particular for $w=2 \operatorname{LCM}(1, \ldots, m)$ for which we know that $\|\cdot\|_{G}^{w} \in S^{w}(G)$ by proposition 7. Using the formula (37) for the modified gauge norm and (39) for the expression of the radial field in exponential coordinates, one then gets (note that $w / \omega_{\ell} \in 2 \mathbb{N}^{*}$ ):

$$
\forall x \in \mathfrak{g} \simeq \mathbb{R}^{q}, \quad \tilde{R}\left(\|x\|_{\mathfrak{g}}^{w}\right)=\sum_{\ell=1}^{q} \omega_{\ell} x_{\ell} \cdot \frac{w}{\omega_{\ell}} x_{\ell}^{\frac{w}{\omega_{\ell}}-1}=w\|x\|_{\mathfrak{g}}^{w}
$$

and thus $\lambda(g)=1$ for any $g \in G$.

\subsection{Adjoints}

Proposition 10 For the $L^{2}(G)$ scalar product, the adjoint vector field to $R$ is

$$
R^{*}(g)=-Q-R(g) .
$$

Proof. The proof is simplest in exponential coordinates, using (39) and (5):

$$
\forall x \in \mathfrak{g} \simeq \mathbb{R}^{q}, \quad \tilde{R}(x)+\tilde{R}^{*}(x)=\operatorname{div} \tilde{R}=\sum_{\ell=1}^{q} \omega_{\ell}=Q .
$$

One can also prove this formula directly, using (42) and (30):

$$
\forall g \in \mathfrak{G}, \quad R(g)+R^{*}(g)=\sum_{\ell} \partial_{\ell}\left(\sigma_{\ell}\right)+\sum_{\ell, \ell^{\prime}} \zeta_{\ell, \ell^{\prime}} \cdot\left(\partial_{\ell^{\prime}} \sigma_{\ell}\right)+\sigma_{\ell} \cdot\left(\partial_{\ell^{\prime}} \zeta_{\ell, \ell^{\prime}}\right) .
$$

In this sum, according to a remark that follows (30), the index $\ell^{\prime}$ is restricted to $\ell^{\prime}>n_{\omega_{\ell}}$ and, in particular, the definition (3) then implies $\omega_{\ell^{\prime}}>\omega_{\ell}$. Now thanks to the remark that follows proposition 8 , one can claim that the variable $x_{\ell}$ does not appear in the second part of $\sigma_{\ell}$, thus its derivative reads

$$
\partial_{\ell}\left(\sigma_{\ell}\right)=\omega_{\ell}
$$

For a similar reason, $\partial_{\ell^{\prime}} \sigma_{\ell}=0$ for $\omega_{\ell^{\prime}}>\omega_{\ell}$. One observes also that in (31), each $\ell_{i}$ involved in the expression of $\zeta_{\ell, \ell^{\prime}}$ must satisfy $\omega_{\ell_{i}}<\omega_{\ell^{\prime}}$. In particular, $\partial_{\ell^{\prime}} \zeta_{\ell, \ell^{\prime}}=0$. One concludes using (5).

The next property checks that left-invariant vector fields on a Carnot group are divergence-free.

Proposition 11 For the $L^{2}(G)$ scalar product, the adjoint vector field to $Y_{\ell}^{L}$ is $-Y_{\ell}^{L}$. In particular, for any smooth function $\psi$ on $G$ and any $\ell_{1}, \ldots, \ell_{n} \in\{1, \ldots, q\}$ :

$$
\int_{G}\left[Y_{\ell_{1}}^{L}, \ldots,\left[Y_{\ell_{n-1}}^{L}, Y_{\ell_{n}}^{L}\right]\right] \psi(g) \cdot \psi(g) d g=0 .
$$


Proof. The second "computational" proof of the previous proposition (the one based on (30)) also ensures that

$$
\partial_{\ell^{\prime}} \zeta_{\ell, \ell^{\prime}}=0
$$

when $\omega_{\ell^{\prime}}>\omega_{\ell}$ and therefore $\left(Y_{\ell}^{L}\right)^{*}=-Y_{\ell}^{L}$. As the commutator of two antisymmetric operators is also an antisymmetric one, the second statement follows immediately.

\subsection{A density result}

The following density result can be proved by a scaling argument.

Proposition 12 The space $\mathcal{D}(G \backslash\{e\})$ of $C^{\infty}$ functions, compactly supported outside the origin, is dense in $H^{s}(G)$ for any $0 \leq s<Q / 2$.

Proof. One can use a Hilbert space approach based on scaling and Schwartz's theorem for distributions. Let us assume additionally that $s \in \mathbb{N}$ and consider a function $u \in H^{s}(G)$ that is orthogonal to any $\phi \in \mathcal{D}(G \backslash\{e\})$, i.e.:

$$
(u, \phi)_{s}=\sum_{|\gamma| \leq s} \int_{G} \nabla_{G}^{\gamma} u(g) \cdot \nabla_{G}^{\gamma} \phi(g) d g=0 .
$$

Integrating by parts (using proposition 11 and the notation $\gamma^{*}$ for the multi-index $\gamma$ in reverse order) reads:

$$
\sum_{|\gamma| \leq s}(-1)^{|\gamma|} \int_{G} \nabla_{G}^{\gamma^{*}} \nabla_{G}^{\gamma} u(g) \cdot \phi(g) d g=0
$$

For fractional values of $s$, one would replace $\nabla_{G}^{\gamma^{*}} \nabla_{G}^{\gamma}$ by a fractional power of the sub-Laplacian (24) and what follows would go unchanged. Schwartz's theorem implies that the distributional support of

$$
v=\sum_{|\gamma| \leq s}(-1)^{|\gamma|} \nabla_{G}^{\gamma^{*}} \nabla_{G}^{\gamma} u
$$

is reduced to the single point $\{e\}$ and thus $v=\sum(-1)^{|\alpha|} c_{\alpha} \partial^{\alpha} \delta$ where $\delta$ is the Dirac function at the origin. As $v$ is at most a $2 s^{\text {th }}$ horizontal derivative of $u$, one has $v \in H^{-s}(G)$ and in particular for any test function $\psi \in \mathcal{D}(G)$ :

$$
\left|\int_{G} v(g) \psi(g) d g\right|^{2} \leq C \sum_{|\gamma| \leq s} \int_{G}\left|\nabla_{G}^{\gamma} \psi(g)\right|^{2} d g .
$$

The constant $C$ does not depend on the support of $\psi$ because $\operatorname{supp} v \subset\{e\}$. In particular, one can apply this inequality to the dilations $\psi(r g)$ for any $r>1$ :

$$
\left|\int_{G} v(g) \psi(r g) d g\right|^{2} \leq C \sum_{|\gamma| \leq s} r^{2|\gamma|} \int_{G}\left|\nabla_{G}^{\gamma} \psi(r g)\right|^{2} d g .
$$

thus

$$
\left|\int_{G} v\left(r^{-1} g\right) \psi(g) d g\right|^{2} \leq C \sum_{|\gamma| \leq s} r^{2|\gamma|+Q} \int_{G}\left|\nabla_{G}^{\gamma} \psi(g)\right|^{2} d g .
$$

Finally, one can compute the left-hand side using the homogeneity of the Dirac mass:

$$
\int_{G} v\left(r^{-1} g\right) \psi(g) d g=\sum_{\alpha} c_{\alpha} r^{Q+\sum \alpha_{j} \omega_{j}} \partial^{\alpha} \psi(e) .
$$

Combining both formulas, one gets for any $r>1$ :

$$
\left|\sum_{\alpha} c_{\alpha} r^{Q+\sum \alpha_{j} \omega_{j}} \partial^{\alpha} \psi(e)\right| \leq C r^{Q / 2+s}\|\psi\|_{H^{s}(G)}
$$


and in particular with a suitable choice of $\psi$ and $r \rightarrow \infty$ :

$$
c_{\alpha} \neq 0 \quad \Longrightarrow \quad s \geq \frac{Q}{2}+\sum \omega_{j} \alpha_{j} .
$$

But as $s<Q / 2$, each coefficient $c_{\alpha}$ vanishes, i.e. $v=0$ in $H^{-s}(G)$ and thus using $u \in H^{s}(G)$ as a test function, one infers $u=0$.

Remark - When $Q$ is even and $s=Q / 2 \in \mathbb{N}$, the previous density result still holds. The only change in the proof is to observe that $\delta \notin H^{-Q / 2}(G)$ by exhibiting an example of an unbounded function in $H^{Q / 2}(G)$; the classical example $\log (-\log \|g\|) \psi(g)$ with a sooth cut-off $\psi$ still works. However, when $Q$ is odd, one still has $\delta \notin H^{-Q / 2}(G)$ but the density result fails as it already does in $H^{n+\frac{1}{2}}\left(\mathbb{R}^{2 n+1}\right)$. For more details on this point, see [29].

\subsection{Hardy inequality}

In this final section, let us combine the previous results into a proof of Theorem 1.

Given $f \in H^{s}(G)$ with $s<Q / 2$ and the density result of the previous section, one can assume without restriction that $f$ is compactly supported and that $0 \notin \operatorname{Supp} u$. Next, one will take a smooth cutoff function $\chi: \mathbb{R} \rightarrow[0,1]$ such that $\chi(t)=1$ if $|t|<1 / 2$. For any $\rho_{0}>0$, one has:

$$
\int_{G} \frac{|f(g)|^{2}}{\|g\|_{G}^{2 s}} \leq \int_{G} \frac{|\varphi(g)|^{2}}{\|g\|_{G}^{2 s}}+\left(\frac{2}{\rho_{0}}\right)^{2 s}\|f\|_{L^{2}(G)}^{2} \quad \text { with } \quad \varphi(g)=\chi\left(\frac{\|g\|_{G}}{\rho_{0}}\right) f(g) .
$$

Moreover, one has $\|\varphi\|_{H^{s}(G)} \leq C_{s} \rho_{0}^{-s}\|f\|_{H^{s}(G)}$. Without restriction, one can therefore assume that $f$ (now denoted by $\varphi$ ) is compactly supported in a fixed but arbitrary small annular neighborhood around the origin.

The key of the computation is the following integration by part argument. Using (43), one has

$$
\int_{G} \frac{|\varphi(g)|^{2}}{\|g\|_{G}^{2 s}}=-\frac{1}{2 s} \int_{G} R\left(\frac{1}{\|g\|^{2 s}}\right) \cdot|\varphi(g)|^{2} .
$$

Using proposition 10 and the fact that $\operatorname{supp} \phi$ is an annulus around the origin so that no boundary terms appear:

$$
\left(\frac{Q}{2}-s\right) \int_{G} \frac{|\varphi(g)|^{2}}{\|g\|_{G}^{2 s}}=-\int_{G} \frac{\varphi(g) R(\varphi(g))}{\|g\|^{2 s}} .
$$

According to (42), the radial field can be expressed with left-invariant vector fields:

$$
\left(\frac{Q}{2}-s\right) \int_{G} \frac{|\varphi(g)|^{2}}{\|g\|_{G}^{2 s}}=-\sum_{\ell=1}^{q} \int_{G} \frac{\sigma_{\ell}(x(g)) \varphi(g) Y_{\ell}^{L}(\varphi(g))}{\|g\|^{2 s}} .
$$

What we do next depends on the order of each derivative $Y_{\ell}^{L} \simeq \nabla_{G}^{\omega_{\ell}}$.

Case $m=1$. In the Euclidian case, one uses Cauchy-Schwarz and Young's identity $|a b| \leq \varepsilon a^{2}+\varepsilon^{-1} b^{2}$ with $\varepsilon>0$ small enough so that $s+\varepsilon<Q / 2$, which leads to:

$$
\left(\frac{Q}{2}-s-\varepsilon\right) \int_{G} \frac{|\varphi(g)|^{2}}{\|g\|_{G}^{2 s}} \leq \varepsilon^{-1} C \int_{G} \frac{\left|\nabla_{G} \varphi\right|^{2}}{\|g\|_{G}^{2(s-1)}} .
$$

This proves Hardy's inequality for $s=1$. Interpolation with $L^{2}$ then ensures that the Hardy inequality holds for any $s \in[0,1]$. Finally, the previous estimate provides a bootstrap argument from $s-1$ to $s$ for any $s<Q / 2$. 
Case $m=2$. One uses the Euclidian technique to deal with the horizontal derivatives. For the stratum $V_{2}$, one uses the commutator structure to backtrack one "half" integration by part. More precisely, the right-hand side of (46) becomes for $1 \leq \ell \leq n_{1}$ :

$$
\left|\int_{G} \frac{\sigma_{\ell}(x(g)) \varphi(g) Y_{\ell}^{L}(\varphi(g))}{\|g\|^{2 s}}\right| \leq \varepsilon \int_{G} \frac{|\varphi(g)|^{2}}{\|g\|_{G}^{2 s}}+C_{\varepsilon} \int_{G} \frac{\left|\nabla_{G} \varphi(g)\right|^{2}}{\|g\|_{G}^{2(s-1)}}
$$

and using (22) for $n_{1}<\ell \leq n_{2}=q$ and proposition 11 (notice the cancellation of the highest order term thanks to the commutator structure):

$$
\begin{aligned}
-\int_{G} \frac{\sigma_{\ell}(x(g))}{\|g\|^{2 s}} \varphi(g) \cdot\left[Y_{\alpha_{1}(\ell)}^{L}, Y_{\alpha_{2}(\ell)}^{L}\right](\varphi(g))= & \int_{G} Y_{\alpha_{1}(\ell)}^{L}\left(\frac{\sigma_{\ell}(x(g))}{\|g\|^{2 s}}\right) \varphi(g) \cdot Y_{\alpha_{2}(\ell)}^{L}(\varphi(g)) \\
& -\int_{G} Y_{\alpha_{2}(\ell)}^{L}\left(\frac{\sigma_{\ell}(x(g))}{\|g\|^{2 s}}\right) \varphi(g) \cdot Y_{\alpha_{1}(\ell)}^{L}(\varphi(g)) .
\end{aligned}
$$

Using the symbol properties of $\|\cdot\|_{G}$ and $\sigma_{\ell}(x(g))$, both terms are bounded in the following way:

$$
\int_{G} \frac{|\varphi(g)| \cdot\left|\nabla_{G} \varphi(g)\right|}{\|g\|_{G}^{2 s-1}} \leq \varepsilon \int_{G} \frac{|\varphi(g)|^{2}}{\|g\|_{G}^{2 s}}+C_{\varepsilon} \int_{G} \frac{\left|\nabla_{G} \varphi(g)\right|^{2}}{\|g\|_{G}^{2(s-1)}} .
$$

One thus gets (47) again and one can conclude the proof just as in the case $m=1$.

Case $m \geq 3$. The additional terms on the right-hand side of (46) correspond to $n_{2}<\ell \leq q$. Thanks to (22), one can express each of them with commutators from the first stratum:

$$
I_{\ell}(\varphi)=-\int_{G} \frac{\sigma_{\ell}(x(g))}{\|g\|^{2 s}} \varphi(g) \cdot\left[Y_{\alpha_{1}(\ell)}^{L}, \ldots,\left[Y_{\alpha_{\omega_{\ell}-1}(\ell)}^{L}, Y_{\alpha_{\omega_{\ell}}(\ell)}^{L}\right]\right](\varphi(g)) .
$$

As in the case $m=2$, the key is to use the commutator structure to put all the derivatives but one on the symbol. More precisely, using proposition 11, one first gets:

$$
I_{\ell}(\varphi)=\frac{1}{2} \int_{G}\left[Y_{\alpha_{1}(\ell)}^{L}, \ldots,\left[Y_{\alpha_{\omega_{\ell}-1}(\ell)}^{L}, Y_{\alpha_{\omega_{\ell}}(\ell)}^{L}\right]\right]\left(\frac{\sigma_{\ell}(x(g))}{\|g\|^{2 s}}\right)|\varphi(g)|^{2} .
$$

Next, one puts the outermost derivative back out onto $\varphi(g)^{2}$ :

$$
I_{\ell}(\varphi)=-\sum_{i=1}^{\omega_{\ell}} \int_{G} W_{\ell, i}\left(\frac{\sigma_{\ell}(x(g))}{\|g\|^{2 s}}\right) \varphi(g) \cdot Y_{\alpha_{i}(\ell)}^{L} \varphi(g)
$$

where each $W_{\ell, i}$ is a derivative of order $\omega_{\ell}-1$. The symbol property $\sigma_{\ell}(x(g)) \in S^{\omega_{\ell}}(G)$ given by proposition 8 ensures that

$$
\left|W_{\ell, i}\left(\frac{\sigma_{\ell}(x(g))}{\|g\|^{2 s}}\right)\right| \leq \frac{C_{\ell, i, s}}{\|g\|_{G}^{2 s-1}} .
$$

Again, one gets

$$
\left|I_{\ell}(\varphi)\right| \leq \varepsilon \int_{G} \frac{|\varphi(g)|^{2}}{\|g\|_{G}^{2 s}}+C_{\varepsilon} \int_{G} \frac{\left|\nabla_{G} \varphi(g)\right|^{2}}{\|g\|_{G}^{2(s-1)}}
$$

and (47) holds once more. As in the case $m=1$, one thus gets the Hardy inequality for $s=1$. Then, by interpolation with $L^{2}(G)$, one gets it for $s \in[0,1]$. Finally, using (47) iteratively, one can collect it for any $s<Q / 2$.

Remark - When $Q$ is odd and $s \in \mathbb{N}$, one can always take $\varepsilon$ small enough so that $\frac{Q}{2}-s-\varepsilon \neq 0$ in (47). The previous iteration argument thus proves the Hardy inequality (6) for any $s \in \mathbb{N}$, but the result is then only valid for functions that belong to the $H^{S}(G)$-closure of smooth compactly supported functions whose support avoids the origin. 


\subsection{Homogeneous Hardy inequality}

One can slightly improve (6) by using a simple scaling argument. For simplicity, we will only spell out the procedure for $s \in \mathbb{N}$ and $0 \leq s<Q / 2$ though it would also work for fractional values of $s$ if $\nabla_{G}^{s}$ was replaced by the corresponding power $\mathcal{L}_{G}^{s / 2}$ of the subelliptic Laplace operator (24).

Theorem 13 For $0 \leq s<Q / 2$, the following homogeneous inequality holds:

$$
\forall f \in H^{s}(G) \quad \int_{G} \frac{|f(g)|^{2}}{\|g\|^{2 s}} d g \leq 2 C_{s}\left\|\nabla_{G}^{s} f\right\|_{L^{2}(G)}^{2} .
$$

Proof. Let us indeed apply (6) to the function $f\left(r^{-1} g\right)$. After the change of variable $g=r \bar{g}$, one gets:

$$
r^{Q-2 s} \int_{G} \frac{|f(\bar{g})|^{2}}{\|\bar{g}\|^{2 s}} d \bar{g} \leq C_{s} \sum_{|\alpha| \leq s} r^{Q-2|\alpha|} \int_{G}\left|\nabla_{G}^{\alpha} f(\bar{g})\right|^{2} d \bar{g}
$$

which, for $r<1$, can be further simplified into:

$$
\int_{G} \frac{|f(\bar{g})|^{2}}{\|\bar{g}\|^{2 s}} d \bar{g} \leq C_{s} \sum_{|\alpha|=s} \int_{G}\left|\nabla_{G}^{\alpha} f(\bar{g})\right|^{2} d \bar{g}+C_{s} r^{2} \sum_{|\alpha| \leq s-1} \int_{G}\left|\nabla_{G}^{\alpha} f(\bar{g})\right|^{2} d \bar{g} .
$$

Choosing

$$
r^{2}=\min \left\{1 ; \frac{\left\|\nabla_{G}^{s} f\right\|_{L^{2}(G)}^{2}}{\|f\|_{H^{s-1}(G)}^{2}}\right\}
$$

instantly leads to (48).

Remark - It would have been tempting to try using (45)-(46) without digging further in the commutator structure to get

$$
\int_{G} \frac{|f(g)|^{2}}{\|g\|_{G}^{2 s}} d g \leq \int_{G}|f(g)|^{2} d g+\left(\frac{Q}{2}-s-\varepsilon\right)^{-1} \sum_{\ell=1}^{q} \int_{G} \frac{\left|Y_{\ell}^{L}((\chi f)(g))\right|^{2}}{\|g\|^{2\left(s-\omega_{\ell}\right)}} d g .
$$

For $s=1$, it gives a Hardy inequality with $\|f\|_{H^{m}(G)}^{2}$ on the right-hand side. However, a scaling argument is then not sufficient to deduce the correct one, either (6) or (48). Indeed, one would simultaneously need to let $r \rightarrow \infty$ and $r \rightarrow 0$ to get rid of the superfluous derivatives without letting the lower-order $L^{2}(G)$ term get in the way, which is overall impossible.

\section{A remark about the case of general hypoelliptic vector fields}

For general families of vector fields that satisfy a Hörmander condition of step $m$, the technique of proving the Hardy inequality by integration by part works, but possibly with some restrictions.

\subsection{A counter-example to the symbol property of the gauge}

The main objection is the following one. When the group structure is discarded, the fact that one can chose a gauge pseudo-norm in a symbol class of order 1 can fail.

For example, the family

$$
Z_{1}=\partial_{1}+x_{1} \partial_{3}, \quad Z_{2}=\partial_{2}+x_{4} \partial_{3}+x_{5} \partial_{4} \quad \text { and } \quad Z_{3}=\partial_{5}
$$

is uniformly of rank 3 in $\mathbb{R}^{5}$ and satisfies a uniform Hörmander bracket condition of step 3 :

$$
\partial_{4}=\left[Z_{3}, Z_{2}\right], \quad \partial_{3}=\left[\left[Z_{3}, Z_{2}\right], Z_{2}\right] .
$$


However, the "natural" gauge:

$$
\rho=\left(\left|x_{1}\right|^{12}+\left|x_{2}\right|^{12}+\left|x_{3}\right|^{4}+\left|x_{4}\right|^{6}+\left|x_{5}\right|^{12}\right)^{1 / 12}
$$

is not a symbol of order 1 because $\left|Z_{1} \rho\right| \geq c \rho^{-1}$ along $x_{1}^{3}-x_{3}=x_{2}=x_{4}=x_{5}=0$. Luckily, for this particular family, the change of variable $y_{3}=x_{3}-\frac{1}{2} x_{1}^{2}$ and $y_{i}=x_{i}(i \neq 3)$ transforms the family into $Z_{1}^{\prime}=\partial_{y_{1}}, Z_{2}^{\prime}=\partial_{y_{2}}+y_{4} \partial_{y_{3}}+y_{5} \partial_{y_{4}}$ and $Z_{3}^{\prime}=\partial_{y_{5}}$ and for this new family, the associated gauge is a symbol of order 1 .

In [29, chap. 7], it was shown that up to a Hörmander condition of step 3, one can always modify the gauge by a local diffeomorphism to restore the symbol property. However, the same question for a family of vector fields that satisfy a Hörmander condition of step 4 or higher is still open. For the convenience of the reader, we will recall here briefly the key points of the discussion (and clarify the redaction), as this result was written in French and never published.

\subsection{Regular hypoelliptic vector fields of step $m$}

Let us consider a family $\mathfrak{X}=\left(X_{\ell}\right)_{1 \leq \ell \leq n_{1}}$ of vector fields on some smooth open set $\Omega \subset \mathbb{R}^{q}$ and

$$
\forall x \in \Omega, \quad W_{k}(x)=\operatorname{Span}\left(X_{i}(x), \ldots,\left[X_{j_{1}}, \ldots,\left[X_{j_{k-1}}, X_{j_{k}}\right]\right](x)\right) .
$$

One assumes that $\underline{x} \in \Omega$ is a regular Hörmander point, i.e. that $n_{k}=\operatorname{dim} W_{k}$ is constant near $\underline{x}$ and that $n_{m}=q$ for some finite integer $m \geq 2$.

Remark - At the origin of a Carnot group (2), one would have $W_{k}(e)=\oplus_{j=1}^{k} V_{j}$.

Next, one introduces a local basis of vector fields $\left(Y_{\ell}(x)\right)_{1 \leq \ell \leq q}$, adapted to the stratification, i.e. $Y_{\ell}(x) \in W_{\omega_{\ell}}(x)$ where for each $\ell$, the weight $\omega_{\ell} \in\{1, \ldots, m\}$ is defined by (3). For simplicity, one will now restrict $\Omega$ to be a bounded and small enough neighborhood of $\underline{x}$ on which all those properties hold. The analog of horizontal derivatives is the family:

$$
\nabla_{\mathfrak{X}}=\left(Y_{1}, \ldots, Y_{n_{1}}\right) \text {. }
$$

A local coordinate system $\left(x_{\ell}\right)_{1 \leq \ell \leq q}$ is said to be adapted to the commutator structure of the vector fields $\mathfrak{X}$ near $\underline{x}$ if the dual basis $\left(\partial_{\ell}\right)_{1 \leq \ell \leq q}$ satisfies $Y_{\ell}(\underline{x})=\partial_{\ell}$.

Remark - Let us point out that adapted coordinates are not necessarily privileged in the sense of A. Bellaiche [9] and M. Gromov [16]: the point of coordinates $\left(x_{\ell}\right)_{1 \leq \ell \leq q}$ does not necessarily match with the image of $\underline{x}$ under the composite action of the flows $e^{x_{\ell} Y_{\ell}}$ (for some predetermined order of composition).

In an adapted coordinate system, the gauge is defined by:

$$
\rho(x)=\left(\sum_{\ell=1}^{q}\left|x_{\ell}\right|^{w / \omega_{\ell}}\right)^{1 / w}
$$

where $w=2 \operatorname{LCM}(1, \ldots, m)$ and the basis of vector fields and their commutators satisfy

$$
\forall \ell \in\{1, \ldots, q\}, \quad Y_{\ell}(x)=\partial_{\ell}+\sum_{\ell^{\prime}=1}^{q} \zeta_{\ell, \ell^{\prime}}(x) \partial_{\ell^{\prime}} .
$$

One obviously has $\left|x_{\ell}\right| \leq \rho(x)^{\omega_{\ell}}$ and, using a Taylor expansion, $\zeta_{\ell, \ell^{\prime}}\left(x_{0}\right)=0$ implies $\left|\zeta_{\ell, \ell^{\prime}}(x)\right| \leq C \rho(x)$. However, for derivatives, one can only claim that $\nabla_{\mathfrak{X}}^{\gamma} x_{\ell}$ and $\nabla_{\mathfrak{X}}^{\gamma} \zeta_{\ell, \ell^{\prime}}$ are bounded when $|\gamma| \geq 1$. 


\subsection{A positive result for hypoelliptic fields of step 2}

Theorem 14 Let us consider a family of vector fields and $\underline{x} \in \Omega$ a regular Hörmander point of step $m=2$. Then for any adapted coordinate system, the gauge $\rho$ satisfies

$$
\left|\nabla_{\mathfrak{X}}^{\gamma} \rho\right| \leq C_{\gamma} \rho^{1-|\gamma|}
$$

in the neighborhood of $\underline{x}$, for any multi-index $\gamma$.

Proof. For $\gamma=0$, the estimate (53) comes from the fact that $\rho$ is smooth and vanishes at the origin and thus admits a Taylor expansion at the origin that is locally bounded by $\sum\left|x_{\ell}\right|$ and thus by $\rho$. For $|\gamma|=1$, the computation is actually explicit:

$$
\nabla_{\mathfrak{X}} \rho=\frac{1}{\rho^{3}}\left(x_{\ell}^{3}+\sum_{\ell^{\prime} \leq n_{1}} \zeta_{\ell, \ell^{\prime}} x_{\ell^{\prime}}^{3}+\frac{1}{2} \sum_{\ell^{\prime}>n_{1}} \zeta_{\ell, \ell^{\prime}} x_{\ell^{\prime}}\right)_{1 \leq \ell \leq n_{1}} .
$$

In the parenthesis, the first term is locally bounded by $\rho^{3}$, the second by $\rho^{4}$ and the last one again by $\rho^{3}$, thus $\nabla_{\mathfrak{X}} \rho \in L^{\infty}(\Omega)$ provided $\Omega$ is small enough. To deal with the higher-order derivatives, let us introduce the class $\mathcal{P}_{n}$ of homogeneous polynomials of $x_{\ell}$ and $\zeta_{\ell, \ell^{\prime}}$ with smooth coefficients, i.e.

$$
\sum_{\alpha, \beta} x_{1}^{\alpha_{1}} \ldots x_{q}^{\alpha_{q}} \zeta_{1,1}^{\beta_{1,1}} \zeta_{1,2}^{\beta_{1,2}} \ldots \zeta_{q, q}^{\beta_{q, q}} f_{\alpha, \beta}(x)
$$

where $f_{\alpha, \beta} \in C^{\infty}(\Omega)$ and $\sum \alpha_{i} \omega_{i}+\sum \beta_{j, j^{\prime}}=n$. For $n \leq 0$, one sets $\mathcal{P}_{n}=C^{\infty}(\Omega)$. With Leibnitz formula, one checks immediately that:

$$
\partial_{\ell}\left(\mathcal{P}_{n}\right) \subset \begin{cases}\mathcal{P}_{n-1}+\mathcal{P}_{n} & \text { if } \ell \leq n_{1} \\ \mathcal{P}_{n-2}+\mathcal{P}_{n-1}+\mathcal{P}_{n} & \text { if } \ell>n_{1}\end{cases}
$$

thus $\nabla_{\mathfrak{X}}\left(\mathcal{P}_{n}\right) \subset \mathcal{P}_{n-1}+\mathcal{P}_{n}+\mathcal{P}_{n+1}$. Moreover, for $m \geq n$, any expression in $\mathcal{P}_{m}$ is locally bounded by $C \rho^{n}$ for some constant $C$. We have shown above that $\nabla_{\mathfrak{X}} \rho \in \rho^{-3} \cdot\left(\mathcal{P}_{3}+\mathcal{P}_{4}\right)$. One then gets recursively on $k=|\gamma|$ that $\nabla_{\mathfrak{X}}^{\gamma} \rho$ is a linear combination of expressions

$$
\frac{\mathcal{P}_{m}}{\rho^{n+k-1}}
$$

with $m \geq n$ and is thus locally bounded by $C \rho^{1-k}$.

Remark - One has $\mathcal{P}_{n} \subset \mathcal{P}_{n-2}$. However, for $\ell>n_{1}$, one has $x_{\ell}^{2} \in \mathcal{P}_{4} \cap \mathcal{P}_{2}$ but $x_{\ell}^{2} \notin \mathcal{P}_{3}$.

\subsection{Two positive results for hypoelliptic fields of step $m \geq 3$}

Let us now revert to the case of a general value for $m$. As pointed out at the beginning of this section, one can find a counter-example of a family of vector fields, a regular Hörmander point of step $m=3$ and an adapted coordinate system for which (53) fails. If we tried to run the previous proof, the failure point would be that

$$
\partial_{\ell^{\prime}}\left(\mathcal{P}_{n}\right) \subset \mathcal{P}_{n-\omega_{\ell^{\prime}}}+\ldots+\mathcal{P}_{n-1}+\mathcal{P}_{n}
$$

When computing $\nabla_{\mathfrak{X}}\left(\mathcal{P}_{n}\right)$, the multiplication by $\zeta_{\ell, \ell^{\prime}} \in \mathcal{P}_{1}$ is then not able to compensate for the loss when $\omega_{\ell^{\prime}} \geq 3$. The profound reason is that our knowledge about the way $\zeta_{\ell, \ell^{\prime}}$ vanishes at the origin is too weak.

Definition - A coordinate system adapted to the commutator structure of the vector fields $\mathfrak{X}$ near a regular Hörmander point $\underline{x}$ of step $m$ is called well-adapted if

$$
\forall \ell \in\left\{1, \ldots, n_{1}\right\}, \quad \forall \ell^{\prime} \in\{1, \ldots, q\}, \quad\left|\nabla_{\mathfrak{X}}^{\gamma} \zeta_{\ell, \ell^{\prime}}\right| \leq C_{\gamma} \rho^{\left(\omega_{\ell^{\prime}}-1-|\gamma|\right)_{+}}
$$

in a neighborhood of $\underline{x}$. A family of vector fields that satisfies a regular Hörmander condition is called well-structured if it admits a well-adapted coordinate system. 
One can check that in a well-adapted coordinate system, the gauge automatically satisfies (53).

Theorem 15 (If-theorem for arbitrary step $m$ ) Let us consider a family of vector fields and $\underline{x} \in \Omega$ a regular Hörmander point of step $m$. Then for any well adapted coordinate system, the gauge $\rho$ satisfies (53) in the neighborhood of $\underline{x}$.

Proof. The key is to adapt the definition of $\mathcal{P}_{n}$ to capture the enhanced knowledge that we gained about $\zeta_{\ell, \ell^{\prime}}$. Let us define $\widetilde{\mathcal{P}}_{n}$ as the subset of $C^{\infty}(\Omega)$ that consists of homogeneous polynomials with smooth coefficients of $x_{\ell}, \zeta_{\ell, \ell^{\prime}}$ and of the derivatives of $\zeta_{\ell, \ell^{\prime}}$ for which we have estimates, i.e.

$$
\sum_{\alpha, \beta} x_{1}^{\alpha_{1}} \ldots x_{q}^{\alpha_{q}} \zeta_{1,1}^{\beta_{1,1}} \zeta_{1,2}^{\beta_{1,2}} \ldots \zeta_{q, q}^{\beta_{q, q}}\left(\prod_{\gamma}\left(\nabla_{\mathfrak{X}}^{\gamma} \zeta_{1,1}\right)^{\delta_{\gamma ; 1,1}} \ldots\left(\nabla_{\mathfrak{X}}^{\gamma} \zeta_{n_{1}, q}\right)^{\delta_{\gamma ; n_{1}, q}}\right) f_{\alpha, \beta, \delta}(x)
$$

where $f_{\alpha, \beta, \delta} \in C^{\infty}(\Omega), \gamma$ denotes multi-indices of length $|\gamma| \geq 1$ and

$$
\sum_{1 \leq i \leq q} \alpha_{i} \omega_{i}+\sum_{j=1}^{q} \sum_{j^{\prime}=1}^{q} \beta_{j, j^{\prime}}\left(\omega_{j^{\prime}}-1\right)+\sum_{|\gamma| \geq 1} \sum_{j=1}^{n_{1}} \sum_{j^{\prime}=1}^{q} \delta_{\gamma ; j, j^{\prime}}\left(\omega_{j^{\prime}}-1-|\gamma|\right)_{+}=n .
$$

Note that only the factors for which $\omega_{j^{\prime}}-1-|\gamma|>0$ are significant; the others can simply be tossed into $f_{\alpha, \beta, \delta}$. For $n \leq 0$, one sets again $\widetilde{\mathcal{P}}_{n}=C^{\infty}(\Omega)$. We also introduce the linear span

$$
\widetilde{\mathcal{P}}_{n}^{+}=\sum_{m \geq n} \widetilde{\mathcal{P}}_{m}
$$

Using the Leibnitz formula, $\nabla_{\mathfrak{X}}\left(x_{\ell}\right) \in \widetilde{\mathcal{P}}_{\omega_{\ell}-1}$ and $\nabla_{\mathfrak{X}}\left(\widetilde{\mathcal{P}}_{n}\right) \subset \widetilde{\mathcal{P}}_{n-1}^{+}$. One also has

$$
\nabla_{\mathfrak{X}} \rho=\frac{\nabla_{\mathfrak{X}}\left(\rho^{w}\right)}{w \rho^{w-1}} \in \rho^{-(w-1)} \cdot \widetilde{\mathcal{P}}_{w-1}^{+}
$$

and recursively (note that $\rho^{w} \in \widetilde{\mathcal{P}}_{w}$ allows one to convert $\widetilde{\mathcal{P}}_{0}$ into $\rho^{-w} \cdot \widetilde{\mathcal{P}}_{w}$ )

$$
\nabla_{\mathfrak{X}}^{\gamma} \rho \in \sum_{n \geq 1} \frac{\mathcal{P}_{n}^{+}}{\rho^{n+|\gamma|-1}}
$$

from which (53) follows immediately.

The previous "abstract" theorem does not presume on the existence of a well-adapted coordinate system. However, when $m \leq 3$, it can actually be made to work.

Theorem 16 Any family of vector fields that satisfies a regular Hörmander condition of step $m \leq 3$ admits at least one well-adapted coordinate system. It is therefore well-structured.

Proof. For $m=1$ and 2, any adapted coordinate system is well-adapted. Let us thus focus on $m=3$ and use the previous notations. Writing down the Taylor expansion of the coefficients for $\ell \leq n_{1}$ :

$$
\zeta_{\ell, \ell^{\prime}}(x)=\sum_{i \leq n_{1}}\left(\frac{\partial \zeta_{\ell, \ell^{\prime}}}{\partial x_{i}}(\underline{x})\right) x_{i}+O\left(\rho^{2}\right),
$$

it appears that, for $m=3$, a coordinate system is well-adapted if and only if

$$
\forall \ell_{1}, \ell_{2} \in\left\{1, \ldots, n_{1}\right\}, \quad \forall \ell_{3} \in\left\{n_{2}+1, \ldots, q\right\}, \quad \frac{\partial \zeta_{\ell_{1}, \ell_{3}}}{\partial x_{\ell_{2}}}(\underline{x})=0 .
$$


Let us compute the following commutator:

$$
\left[Y_{\ell_{1}}, Y_{\ell_{2}}\right]=\sum_{\ell=1}^{q}\left(\frac{\partial \zeta_{\ell_{2}, \ell}}{\partial x_{\ell_{1}}}-\frac{\partial \zeta_{\ell_{1}, \ell}}{\partial x_{\ell_{2}}}\right) \partial_{\ell}
$$

At the point $\underline{x}$, the terms corresponding to $\ell>n_{2}$ must belong to $W_{2}(\underline{x})$ and thus vanish, therefore:

$$
\forall \ell_{1}, \ell_{2} \in\left\{1, \ldots, n_{1}\right\}, \quad \forall \ell_{3} \in\left\{n_{2}+1, \ldots, q\right\}, \quad \frac{\partial \zeta_{\ell_{1}, \ell_{3}}}{\partial x_{\ell_{2}}}(\underline{x})=\frac{\partial \zeta_{\ell_{2}, \ell_{3}}}{\partial x_{\ell_{1}}}(\underline{x}) .
$$

One can now define a new coordinate system $\left(y_{\ell}\right)_{1 \leq \ell \leq q}$ whose dual basis satisfies

$$
\frac{\partial}{\partial y_{\ell}}=\frac{\partial}{\partial x_{\ell}}+\mathbf{1}_{\ell \leq n_{1}} \sum_{\ell^{\prime}>n_{2}} \sum_{i \leq n_{1}}\left(\frac{\partial \zeta_{\ell, \ell^{\prime}}}{\partial x_{i}}(\underline{x})\right) x_{i} \cdot \frac{\partial}{\partial x_{\ell^{\prime}}} .
$$

This coordinate system is (locally) well defined because the fields $\frac{\partial}{\partial y_{\ell}}$ commute with each other thanks to (56). By construction, this coordinate system satisfies (55) and is therefore a well-adapted one.

Remark - The generalization of theorem 16 for $m \geq 4$ is an open question. One can check that a coordinate system is well-adapted if and only if

$$
\frac{\partial^{\alpha} \zeta_{\ell, \ell^{\prime}}}{\partial x_{1}^{\alpha_{1}} \ldots \partial x_{q}^{\alpha_{q}}}(\underline{x})=0
$$

for any indices such that $\omega_{\ell}=1, \omega_{\ell^{\prime}} \geq 3$ and $\sum_{i=1}^{q} \omega_{i} \alpha_{i} \leq \omega_{\ell^{\prime}}-2$. However, for $m \geq 4$, it is not clear whether the regular Hörmander assumption of step $m$ is enough to ensure that the vector fields

$$
\frac{\partial}{\partial y_{\ell}}=\frac{\partial}{\partial x_{\ell}}+\mathbf{1}_{\ell \leq n_{1}} \sum_{\ell^{\prime}>n_{2}}\left(\sum_{\sum \alpha_{i} \omega_{i} \leq \omega_{\ell^{\prime}}-2}\left(\frac{\partial^{\alpha} \zeta_{\ell, \ell^{\prime}}}{\partial x_{1}^{\alpha_{1}} \ldots \partial x_{q}^{\alpha_{q}}}(\underline{x})\right) x_{1}^{\alpha_{1}} \ldots x_{q}^{\alpha_{q}} \cdot \frac{\partial}{\partial x_{\ell^{\prime}}}\right)
$$

commute with each other.

\subsection{From the symbol property of the gauge to Hardy inequality}

For well-structured families of vector fields, symbols of class $S^{k}(\mathfrak{X} ; \rho)$ are functions $f$ such that

$$
\left|\nabla_{\mathfrak{X}}^{\gamma} f(x)\right| \leq C_{\gamma} \rho(x)^{(k-|\gamma|)_{+}}
$$

in a neighborhood of $\underline{x}$, for any multi-index $\gamma$. Once the symbol property is established for the gauge, the path that leads to the Hardy inequality is open. The key (see [29, chap. 7]) is to define a "radial" vector field that admits both expressions:

$$
R(x)=\sum_{\ell=1}^{q} \sigma_{\ell}(x) Y_{\ell}(x)=\sum_{k=1}^{q}\left(\omega_{k} x_{k}+\widetilde{\sigma}_{k}(x)\right) \partial_{k}
$$

in well-adapted coordinates, with $\sigma_{\ell} \in S^{\omega_{\ell}}(\mathfrak{X} ; \rho)$ and $\widetilde{\sigma}_{k} \in S^{\omega_{k}+1}(\mathfrak{X} ; \rho)$. One can then check that

$$
\operatorname{div} R=Q+O(\rho) \quad \text { and } \quad \lambda=\frac{R \rho}{\rho} \quad \text { satisfies }\left\{\begin{array}{l}
\lambda(x)=1+O(\rho), \\
R \lambda=O(\rho) .
\end{array}\right.
$$

The computations of $\S 2.5$ can then be carried out in a small enough neighborhood of $\underline{x}$. 


\section{References}

[1] Adimurthi, A. Mallick. A Hardy type inequality on fractional order Sobolev spaces on Heisenberg group. Ann. Sc. Norm. Super. Pisa Cl. Sci. (5) 18 (2018), no. 3, 917-949.

[2] L. Ambrosio, A. Pinamonti, G. Speight. Weighted Sobolev spaces on metric measure spaces. J. Reine Angew. Math. 746 (2019), 39-65.

[3] L. Ambrosio, S. Rigot. Optimal mass transportation in the Heisenberg group. J. Funct. Anal. 208 (2004), no. 2, 261-301.

[4] H. Bahouri, J.-Y. Chemin, I. Gallagher. Precised Hardy inequalities on $\mathbb{R}^{d}$ and on the Heisenberg group $\mathbb{H}^{d}$. Sémin. Équ. Dériv. Partielles (2004-2005), Exp. No. XIX, 17 pp.

[5] H. Bahouri, J.-Y. Chemin, C.-J. Xu. Trace and trace lifting theorems in weighted Sobolev spaces. J. Inst. Math. Jussieu 4 (2005), no. 4, 509-552.

[6] H. Bahouri, J.-Y. Chemin, C.-J. Xu. Trace theorem on the Heisenberg group. Ann. Inst. Fourier, 59 (2009), no. 2, 491-514.

[7] H. Bahouri, A. Cohen. Refined Sobolev Inequalities in Lorentz Spaces. J. Fourier. Anal. Appl. 17 (2011), no. $4,662-673$.

[8] H. Bahouri, C. Fermanian, I. Gallagher. Refined inequalities on graded Lie groups. C.R. Math. Acad. Sci. Paris 350 (2012), no. 7-8, 393-397.

[9] A. Bellaïche. The tangent space in sub-Riemannian geometry, in Sub-Riemannian Geometry. Birkhäuser, Progress in Maths, Vol. 144 (1996).

[10] P. Ciatti, M.G. Cowling, F. Ricci. Hardy and uncertainty inequalities on stratified Lie groups. Advances in Mathematics. Vol. 277, 4 (2015), 365-387.

[11] L. D'Ambrosio. Hardy-type inequalities related to degenerate elliptic differential operators. Ann. Sc. Norm. Super. Pisa Cl. Sci. (5) 4 (2005), no. 3, 451-486.

[12] J. Dou, P. Niu, Z. Yuan. A Hardy inequality with remainder terms in the Heisenberg group and the weighted eigenvalue problem. J. Inequal. Appl. 2007. Art. ID 32585, 24 pp.

[13] G.B. Folland, E.M. Stein. Hardy spaces on homogeneous groups. Mathematical Notes, 28. Princeton Univ. Press, 1982.

[14] N. Garofalo, E. Lanconelli. Frequency functions on the Heisenberg group, the uncertainty principle and unique continuation. Ann. Inst. Fourier, 40-2 (1990).

[15] G. Grillo. Hardy and Rellich-type Inequalities for Metrics Defined by Vector Fields. Potential Anal., 18-3 (2003), 187-217.

[16] M. Gromov. Carnot-Carathéodory spaces seen from within, in Sub-Riemannian Geometry. Birkhäuser, Progress in Maths, Vol. 144 (1996).

[17] G.H. Hardy, J.E. Littlewood, G. Pólia. Inequalities. Cambridge Univ. Press, 1934.

[18] L. Hormander. Hypoelliptic second order differential equations. Acta Math., 119 (1967), 147-171.

[19] S. Klarsfeld, J.A. Oteo. The Baker-Campbell-Hausdorff formula an the Convergence of the Magnus Expansion. J. Phys. A : Math. Gen. 22 (1989), 4565-4572.

[20] I. Kombe. Sharp weighted Hardy-type inequalities and uncertainty principle inequalities on Carnot Groups. Commun. Appl. Anal. 14 (2010), no. 2, 251-271.

[21] N. Krugljak, L. Maligranda, L.-E. Persson. The failure of the Hardy inequality and interpolation of intersections. Ark. Mat., 37 (1999), 323-344.

[22] R. Montgomery. A Tour of Subriemannian Geometries. AMS Surveys, 2002.

[23] S. Mustapha, F. Vigneron. Construction of Sobolev spaces of fractional order with sub-Riemannian vector fields. Ann. Inst. Fourier, Vol. 57 (2007), no. 4, 1023-1049.

[24] P. Niu, H. Zhang, Y. Wang. Hardy type and Rellich type inequalities on the Heisenberg group. Proc. Amer. Math. Soc. 129 (2001), no. 12, 3623-3630.

[25] C. Reutenauer. Free Lie algebras. Oxford University Press (1993). 
[26] W. Rossmann. Lie Groups. Oxford Univ. Press, 2002.

[27] M. Ruzhanskya, D. Suraganab. On horizontal Hardy, Rellich, Caffarelli-Kohn-Nirenberg and p-sub-Laplacian inequalities on stratified groups. J. Diff. Equations, 262, no. 3 (2017), 1799-1821.

[28] F. Vigneron. The trace problem for weighted Sobolev spaces over the Heisenberg group. J. Anal. Math. 103 (2007), 279-306.

[29] F. Vigneron. Espaces fonctionnels associés à une famille de champs de vecteurs. PhD Thesis. École Polytechnique, 2006 (178 pages, french).

https : //www.dropbox.com/s/yv9r1rinr1pjxrs/these.pdf 